\title{
Sexuality of young women surviving leukaemia
}

\author{
L-R M Puukko, E Hirvonen, V Aalberg, L Hovi, J Rautonen, M A Siimes
}

\begin{abstract}
This study was designed to assess the sexuality of young women surviving acute leukaemia in childhood or early adolescence. Thirty of 31 survivors were compared with 50 healthy age matched controls. Three methods were used: a self report questionnaire, a face to face interview conducted by a psychiatrist, and a projective psychological test. The age at initiation of dating and sexual activity, the frequency of sexual intercourse, and opinions on sexual behaviour were similar in the two groups. With regard to inner sexuality, however, the survivors differed significantly from the healthy controls. Their images of sexuality were more restrictive, and their attitudes, especially those concerning sexual pleasure, were more negative than those of the controls. Sexual identity among the survivors was less often feminine and more often infantile as compared with the controls. The findings obtained with the three methods of assessment were concordant.

(Arch Dis Child 1997;76:197-202)
\end{abstract}

Keywords: leukaemia; adolescent; sexuality.

Advances in the treatment of childhood cancer have made it possible to identify long term effects in adults. ${ }^{12}$ Both diagnoses and minor compromises regarding quality of life are significant. $^{3}$

Findings on psychological late effects of childhood cancer in survivors are inconclusive. Some studies dealing primarily with the overall psychological adaptation of survivors have shown good adjustment, ${ }^{4-8}$ whereas others suggest psychological difficulties. ${ }^{9-12}$ The discrepancies are possibly due to variability in methodology-for example, measures focusing on psychopathology alone rather than adaptation, biased samples produced by unwillingness of survivors to participate in research protocols, lack of unbiased controls, or grouping erroneous 'cancer survivors' into a single generic group. ${ }^{13-15}$

Various studies have indicated deficiencies in body image as well as alterations in perception of the self in subjects studied after haematological childhood malignancy. ${ }^{15}$ Madan-Swain et al have reported sexual discomfort among adolescent cancer survivors. ${ }^{14}$ Childhood cancer may affect ability to make contact with the opposite sex ${ }^{9}$ and psychosexual development of male adolescents. ${ }^{16}{ }^{17}$ These findings ${ }^{18}$ may be related to the gonadal damage often seen in male survivors. In contrast, ovarian damage is rare after treatment of leukaemia except after total body irradiation in females treated by bone marrow transplantation.

We are not aware of any reports on sexuality of female survivors of childhood cancer. We assumed that experience of childhood cancer may disturb the development of female sexual identity. In addition, a life threatening illness during childhood is likely to be reflected in sexual attitudes and sexual behaviour such as dating, onset of sexual intercourse, and sexual activity.

The present study was designed to investigate the late effects of childhood cancer on sexuality of female cancer survivors. Its purpose was to assess possible changes in sexual identity, sexual attitudes, and sexual behaviour of female cancer survivors when compared with healthy age matched controls. As sexuality is a complex phenomenon, we used four different methods: a self report questionnaire, a semistructured face to face interview, psychiatric evaluation, and a psychological test. To reduce any variability caused by the disease or its treatment we confined our study to leukaemia survivors.

\section{Methods}

PATIENTS

This population based study comprised all female survivors of acute leukaemia from southern and central Finland diagnosed and treated before 1986 at the Children's Hospital, University of Helsinki, who were over 16 years of age, in complete remission, and off therapy for at least one year at the time of the study. These survivors were identified from off therapy registry records. Of 31 survivors, 30 participated in the study. Their mean age at the time of the study was 20.1 (SD 3.7) years. The mean age at diagnosis of acute leukaemia was 8.5 (3.4) years. The mean duration of chemotherapy was 3.2 (1.9) years, and the mean follow up time 8.4 (3.9) years. Seven of the survivors had less than five years follow up after completing therapy, and 23 had five years or more. The patients had been treated according to Scandinavian protocols. ${ }^{19}$ Of the survivors, five had been treated with an allogenic bone marrow transplant (BMT). The 25 conventionally treated survivors were menstruating normally. Their mean age at menarche was 12.8 (range 11.3-15.0) years. At the time of the BMT, two survivors had experienced spontaneous menarche, whereas three were premenarchal. All five needed sex hormone substitution therapy after BMT to promote pubertal development and menstruation. 
Table 1 Sociodemographic characteristics of female leukemia survivors and healthy controls at the time of the study. (Parental educational level was used to describe the social background of the subjects.) The differences between the survivors and the controls were not statistically significant. Values in parentheses are percentages except where indicated otherwise

\begin{tabular}{lll}
\hline & Survivors $(n=30)$ & Controls $(n=50)$ \\
\hline Age (SD) (years) & $20.1(3.7)$ & $20.4(3.9)$ \\
Employment status & $19(63)$ & $34(68)$ \\
$\quad$ Studying & $10(33)$ & $15(30)$ \\
Employed & $1(3)$ & $1(2)$ \\
$\quad$ Unemployed & $17(57)$ & $30(60)$ \\
Domicile & $5(16)$ & $5(10)$ \\
$\quad$ With parents & $5(16)$ & $13(26)$ \\
Alone & $3(10)$ & $2(4)$ \\
With a male partner & $3(10)$ & $3(6)$ \\
Other & $2(7)$ & $1(2)$ \\
Married & $20(67)$ & $34(68)$ \\
Own children & $4(13)$ & $14(28)$ \\
Family background & $6(20)$ & $2(5)$ \\
Intact family & & $6(12)$ \\
$\quad$ Parents divorced & $3(10)$ & $10(20)$ \\
$\quad$ Other & $2(7)$ & $17(34)$ \\
Parental educational level & $13(43)$ & $16(32)$ \\
Academic education & $12(40)$ & \\
$\quad$ Postsecondary education & Vocational training &
\end{tabular}

Table 2 Sexually related attitudes of the survivors $(n=30)$ and the controls $(n=50)$

\begin{tabular}{|c|c|c|c|c|c|c|c|c|c|c|}
\hline \multirow{2}{*}{$\begin{array}{l}\text { Attitudes towards sexually related } \\
\text { items }\end{array}$} & \multicolumn{5}{|c|}{ Survivors (\%) } & \multicolumn{5}{|c|}{ Controls (\%) } \\
\hline & ++ & + & $+/-$ & - & -- & ++ & + & $+/-$ & - & -- \\
\hline $\begin{array}{l}\text { Abortion should be more } \\
\text { difficult to obtain than it is }\end{array}$ & 17 & 17 & 14 & 38 & 14 & 2 & 16 & 10 & 29 & $43^{\star \star \star}$ \\
\hline $\begin{array}{l}\text { Sexual relations between two } \\
\text { people of the same sex should } \\
\text { be allowed }\end{array}$ & 14 & 28 & 31 & 20 & 7 & 55 & 23 & 6 & 10 & $6^{\star \star \star}$ \\
\hline $\begin{array}{l}\text { If I were unfaithful to my } \\
\text { boyfriend/ husband, it would } \\
\text { be fair if he left me }\end{array}$ & 8 & 33 & 33 & 22 & 4 & 10 & 31 & 8 & 43 & 8 \\
\hline $\begin{array}{l}\text { Dating does not lead to sexual } \\
\text { intercourse at my age }\end{array}$ & 3 & 1 & 3 & 29 & 54 & 2 & 2 & 2 & 22 & 72 \\
\hline $\begin{array}{l}\text { Sexual intercourse without love } \\
\text { is acceptable }\end{array}$ & 3 & 29 & 18 & 18 & 32 & 8 & 31 & 6 & 39 & 16 \\
\hline $\begin{array}{l}\text { Sexual intercourse should take } \\
\text { place only within marriage }\end{array}$ & 11 & 3 & 18 & 25 & 43 & 4 & 4 & 0 & 16 & $76^{\star \star \star}$ \\
\hline $\begin{array}{l}\text { In sexual relations the boy/man } \\
\text { should always be the initiator } \\
\text { A temporary sexual relationship } \\
\text { can be happy and satisfy both } \\
\text { partners }\end{array}$ & 0 & 10 & 10 & 52 & 28 & 0 & 8 & 8 & 27 & $57^{\star}$ \\
\hline $\begin{array}{l}\text { Sex magazines should be } \\
\text { forbidden }\end{array}$ & 7 & 21 & 34 & 21 & 17 & 0 & 6 & 20 & 25 & $49^{\star \star \star}$ \\
\hline
\end{tabular}

All the survivors were of Finnish origin, and table 1 presents the demographic characteristics. Five $(17 \%)$ were cohabiting, of whom three were married; two had children.

CONTROL GROUP

The controls were young women who were age matched to the survivors within a year and had no history of serious or long term illness in childhood or early adolescence. We aimed to obtain an identical number of survivors and controls in each age group so enrolled successive previously healthy females operated on for uncomplicated acute appendicitis in the hospitals of the greater Helsinki area, since we assumed that the disease affects every subpopulation similarly. These individuals were invited to participate in the study at discharge. Of the 28 possible subjects, 23 participated about three months after surgery. To increase the number of controls, we also collected in a similar way 27 successive age matched healthy women who attended for routine dental check ups in a local population based health centre in Helsinki. None refused to participate. These two groups were subsequently pooled for statistical purposes, since no significant differences in psychological variables were observed between them. Thus, there were 50 controls. Their mean age at the time of the study was 20.4 (3.9) years, and their mean age at menarche was 13.2 (range 10.9-16.3) years.

All the controls were of Finnish origin; table 1 presents the demographic data. Thirteen $(26 \%)$ were cohabiting, of whom three were married and one had children.

\section{DATA COLLECTION}

In this study, we defined sexuality as including inner sexuality and sexual behaviour such as dating and intercourse, whereas inner sexuality comprises subjective experiences, sexual fantasies, images, attitudes, and sexual identity.

To increase reliability three approaches were used: a self report questionnaire, a clinical psychiatric interview, and a projective psychological test. The subjects were invited to visit the outpatient clinic of the Children's Hospital for the study. After completion of the structured questionnaire, the same female psychiatrist (experienced in dealing with adolescents and adults) interviewed each confidentially under standardised conditions. Finally, the psychological test was carried out. The criteria for scoring were determined before the interviews and tests were conducted. To avoid contamination, the data were not analysed until it had all been obtained.

The study protocol was approved by the ethics committees of the institutes involved. Informed consent was obtained from all participants.

SELF REPORT QUESTIONNAIRE

The structured questionnaire had been designed for a study of sexual attitudes, fears, and behaviour of Finnish adolescents aged 13, 15, and 17 years in 1986 and 1988 (the KISS study). ${ }^{20}{ }^{21}$ On both occasions, the questionnaires were completed by some 3000 young people living in different parts of Finland. After preliminary tests, the most essential questions were further presented to adolescents of the same age in one area every two years; the distributions appeared identical each time, indicating validity and reliability. In addition, the structured questionnaire has been confirmed by focused interviews. ${ }^{22}$

We investigated attitudes to intercourse, abortion, heterosexual and homosexual relations, infidelity, and sex magazines by nine statements on a five point scale ranging from absolute agreement to total disagreement (table 2). As table 3 shows, five questions dealt with fears and five with how often the issue was discussed with friends on a three point scale from never to often. To ensure validity, there was also a question dealing with dating or having been dated in the self report questionnaire.

THE INTERVIEW

The semistructured face to face interview covered a wide range of developmental aspects of 
Table 3. Questions in the self report questionnaire concerning fears and discussions with friends. Every question offered three possible answers: often, seldom, or never

Have you ever been worried about the following items?

Fear of contracting sexually transmitted disease

Fear of contracting HIV

Fear of becoming pregnant

Fear of not finding a sexual partner

Fear of your own sexual deviance

Have you discussed the following matters with your friends during the last 6 months?

Contraception

Sexual intercourse

Sexually transmitted diseases

Becoming pregnant

Masturbation

adolescence. It lasted for 1.5 to 3.0 hours. Questions designed for this study were mostly open-ended with appropriate follow up questions. The order and nature of the questions within each theme were the same for each interview, which started out with a discussion of everyday themes and proceeded to psychologically more relevant aspects and a disease history. Topics included family and peer relationships, pubertal and sexual experiences and behaviour, health and illness concerns, attitudes, ideals, and expectations from life. Although the subjects were encouraged to elaborate on responses, the interviewer did not interpret or suggest possible answers. The psychiatrist was not acquainted with the subjects or their personal histories before the interview. Since experiences and feelings concerning leukaemia were discussed, the survivors and the controls were inevitably distinguished between. Apart from chance comments, experience of illness was not discussed until the end of the interview. The data obtained were divided into two categories: (1) sexual behaviour and experiences recorded per se as reported by the subjects themselves, and (2) psychiatric evaluations.

(1) Data on sexual behaviour and experiences reported by the subjects themselves were collected to obtain information about dating men: initiation of dating and sexual experiences, first experience of sexual intercourse, and frequency of intercourse. We recorded the subjects' experiences of intercourse (mostly enjoyable, seldom enjoyable, or never enjoyable), their own initiative toward intercourse (often, seldom, or never), their ability to express sexual desires (quite openly, with difficulty, or never), and their thoughts on masturbation (accepting, indifferent, or forbidden). Before questions about their own masturbation, the normality and commonness of masturbation were clarified to the subjects. The replies were classified as: have consciously masturbated, unsure about own masturbation, or definitely never masturbated. Finally, answers about the significance of sexuality in adult life were also classified into three categories; at least some significance, unsure, or insignificant.

(2) Psychiatric evaluation. Images of sexuality were classified into three categories: mainly gratifying, indifferent, or restrictive. We used the term gender identity to mean a basic sense of maleness or femaleness. ${ }^{23}{ }^{24}$ In contrast, we used sexual identity to mean elaboration of the concepts of femininity and masculinity as distinct from actual maleness or femaleness, and as personal eroticism expressed not only in actual sexual behaviour, but in fantasies, images, attitudes, and personal interest in sex..$^{23}$ Sexual identity was classified into three categories: feminine, indifferent, or infantile. It was evaluated as follows: feminine if the subject had an age appropriate interest in sexuality and in seeking a love object, and fantasised enjoyment, activity, and feminine identification; as indifferent if the subject had hardly any interest in age appropriate sexuality or in seeking a love object, or if her fantasies were denied, mainly frightening, or included uncertain identification; infantile if sexual impulses were totally denied or provoked severe anxiety.

PSYCHOLOGICAL TEST

Since we focused on in depth issues, a projective test, the Rorschach test, was selected to supplement the data on sexuality. It is possible to evaluate different aspects of personality with standardised scoring and content analysis using this test. It was conducted and analysed independently by the same female psychologist, who was not previously acquainted with the subjects or their personal histories. The test material was not assessed until all data had been obtained. The interpretation of the results was based on scoring by Rapaport's system ${ }^{25}$ and on content analysis. ${ }^{26}{ }^{27}$

The sexual identity revealed by the Rorschach material was classified into four categories: feminine, indifferent, with masculine features, or infantile. Sexual identity was evaluated as feminine if sexuality was integrated and appropriate for age, and as infantile if sexual impulses were totally denied or provoked a shock reaction.

\section{STATISTICS}

The differences between the groups were analysed with the Mann-Whitney U test. Spearman's rank correlation coefficient test was used for correlations.

\section{Results}

SELF REPORT QUESTIONNAIRE

Several differences in attitudes and thoughts about sexual aspects of life were found between the leukaemia survivors and the controls. The survivors thought more often that sexual relationships should take place only within marriage, that temporary relationships do not bring happiness or satisfaction to both partners $(p<0.001)$, and that men should always be the initiators in sexual relations $(p=0.04)$. They also had more disapproving attitudes towards abortion, homosexuality, and sex magazines than the controls $(p<0.001)$. However, in both groups attitudes to intercourse in their own age group, intercourse without love, and their own infidelity were similar (table 2).

Compared with the controls, the survivors less often reported fear of catching any sexually transmitted disease $(14 \% v 52 \%, \mathrm{p}=0.002)$ or HIV in particular (17\% v 35\%, p = 0.02). Fears concerning pregnancy, sexual deviance, 
and not finding a sexual partner were similar in the two groups. The survivors had less often discussed contraception $(66 \% v 96 \%, \mathrm{p}=$ $0.002)$, sexual intercourse $(58 \% v 88 \%, \mathrm{p}=$ $0.008)$, and pregnancy $(80 \% v 94 \%, \mathrm{p}=0.02)$ with their friends than the controls. In contrast, we observed no difference in the frequency of discussions about sexually transmitted diseases or masturbation.

Some $79 \%$ of the survivors and $92 \%$ of the controls reported that they were or had been dating. Exclusion of BMT survivors or subjects under 18 years of age did not change the statistical differences observed in the self report questionnaire.

\section{SUBJECT'S REPORTS AT INTERVIEW}

We found no statistically significant difference in dating between the groups: $75 \%$ of the survivors and $90 \%$ of the controls were or had been dating. The survivors had started dating at a mean age of $15.6(1.5)$ years and the controls at a mean age of 14.9 (2.5) years.

Experience of sexual intercourse was less common among the survivors than among the controls $(52 \% v 76 \%, \mathrm{p}=0.03)$. The mean age at first intercourse was 16 years in both groups. Onset of dating was associated with age at menarche in the controls $(r=0.41, \mathrm{p}=0.004)$, but not in the survivors. Sexual intercourse was not associated with age at menarche. After the first experience of intercourse, individuals in both groups continued to have sexual intercourse with similar frequency. However, differences were also observed between the groups. Only four of the survivors stated that they had usually enjoyed intercourse, as compared with 26 of the controls $(p=0.01)$. Five survivors had sometimes taken the initiative in sexual intercourse compared with 20 of the controls $(p=0.003)$. Two of the survivors and 20 of the controls were able to express their sexual desires to their partners $(p<0.001)$.

Fewer survivors than controls considered masturbation natural or acceptable $(25 \% v$ $75 \%, \mathrm{p}<0.001) ; 23 \%(\mathrm{n}=7)$ of survivors and $83 \%(\mathrm{n}=40)$ of the 48 controls had consciously masturbated $(\mathrm{p}<0.001)$. The survivors attributed less significance to sexuality in adult life $(37 \% v 89 \%, \mathrm{p}=0.001)$

Of the subjects under 18 years of age, $75 \%$ of the survivors and $84 \%$ of the controls were dating and $18 \%$ of the survivors and $58 \%$ of the controls had had sexual intercourse.

The findings were similar in survivors with follow up time less than and more than five years. Exclusion of neither subjects under 18 years of age nor BMT survivors changed the differences.

\section{PSYCHIATRIC EVALUATION}

Comparing survivors with controls, mental images of sexuality were more restrictive (48\% $v 9 \%)$, or indefinite $(38 \% v 27 \%)$ and less often mainly gratifying $(14 \%$ v 64\%, p<0.001). Gender identity was assessed as clearly female in both groups. In contrast, sexual identity was less often feminine $(23 \% v 56 \%)$ and more often indifferent $(54 \% v 38 \%)$ or infantile $(23 \% v 6 \%)$ among survivors than controls $(\mathrm{p}=0.002)$. Sexual identity was associated with dating $(r=0.56, \mathrm{p}<0.001)$ but not with menarche or age at the start of dating. Sexual identity was not associated with age at the time of the study, age at diagnosis, duration of treatment, or follow up time. The findings were similar whether follow up time was less than or more than five years. The statistical differences remained significant after the subjects under 18 years of age or the BMT survivors were excluded from the analyses.

PSYCHOLOGICAL TEST

In the Rorschach test, sexual identity was less often feminine $(38 \% v 58 \%)$, and more often indifferent $(35 \% v 42 \%)$, indifferent with masculine features $(10 \% v 0 \%)$, or infantile $(17 \% v$ $0 \%$ ) among survivors than controls ( $\mathrm{p}=$ $0.002)$. The findings on sexual identity were similar in the Rorschach test and the psychiatric evaluation $(r=0.77, \mathrm{p}<0.001)$. Sexual identity, as assessed from the Rorschach test, was not associated with age at the time of the study, age at diagnosis, duration of treatment, or follow up time. However, sexual identity on Rorschach testing was less often adequate in the seven survivors with a follow up time of less than five years than in the 22 others $(p=0.02)$. Exclusion of the BMT survivors did not change the statistical differences noted.

\section{Discussion}

Our findings suggest that female survivors of childhood leukaemia do not differ from healthy age matched controls with respect to the following aspects of sexual behaviour: age at which dating began, onset and frequency of sexual intercourse, and opinions on sexual behaviour. This may reflect good overall adaptation as previously reported. ${ }^{4-8}$ However, there were also significant differences in behaviour: survivors were less likely to have experienced sexual intercourse, less likely to have initiated intercourse, less likely to masturbate and less likely to have talked with friends about sexual topics. In addition the survivors differed from the controls in their subjective experience of sexuality. The survivors were more restrictive, passive and submissive, and their conscious expectations of sexual pleasure, if any, were less than those of the controls.

In inner sexuality, vulnerability tended to persist. In the psychiatric evaluation only $23 \%$ of the survivors were assessed as having feminine sexual identity. In contrast, $23 \%$ of the survivors compared with $6 \%$ of the controls were assessed as being unable to handle sexual impulses. These results highlight the existence of different subsets among the survivors: those who are coping well and those who continue to suffer from long term psychological effects.

Although on Rorschach testing $38 \%$ of the survivors were evaluated as having feminine sexual identity, the majority of them were also shown to have problems with inner sexuality. Of the survivors, $17 \%$ were assessed as having infantile sexual identity, which suggests immaturity in psychosexual development. Neither age at the time of the study nor post-treatment time were associated with sexual identity findings, 
whether based on psychiatric evaluation or on the test result. Thus passage of time does not seem to help the survivors with these problems.

Our results also suggest that, despite psychological vulnerability, many female survivors start dating and sexual activity at the same age as healthy female adolescents. The previous reports of sexual discomfort and a desire to appear 'supernormal' among cancer survivors ${ }^{14}$ support our speculation that the survivors might hasten to have sexual relations to prove their normality or fertility. Our results suggest that this tendency to emphasise normality may mask the impairments of sexual identity observed on psychiatric evaluation and Rorschach testing.

Discussions with peers help young people to adapt themselves to their own sexual needs and desires. ${ }^{28}$ The survivors, however, seem to derive less support from their friends in their psychological adaptation to pubertal changes than the healthy controls.

In this study the survivors less often considered masturbation as normal and they consciously masturbated strikingly less often than the controls. Absence of conscious masturbation in female adolescents does not necessarily indicate general problems in sexuality. ${ }^{28}$ However, masturbation is a way in which adolescents adapt to their sexual body. Our findings suggest that the survivors lack interest in their own sexual body and its functions. A plausible explanation is that experience of life threatening illness may have left them feeling that their body is somehow defective. This is supported by previous studies in which the majority of survivors had impaired body image and their previous cancer was felt as a threat. ${ }^{92}$

Sexual identity and sexuality develop differently in males and females. ${ }^{30}$ In two previous studies, male survivors of leukaemia were shown to have difficulties with sexuality. ${ }^{16}$ Our findings suggest that female survivors succeed better in overt sexual behaviour than male survivors. However, females are capable of sexual relationships irrespective of the psychological significance of sexuality, whereas the sexual relationships of males require closer integration of sexuality. This phenomenon of precocity in the onset of sexual life has been documented among female adolescents. ${ }^{31}$ Thus, although the better overt sexual behaviour of female survivors may give a more positive view of sexuality in females than in males, our findings indicate vulnerability at the level of subjective sexual experiences.

Our survivors represented a population based series, since all the children with acute leukaemia from south and central Finland are treated in the same hospital and all the survivors except one participated. The survivor series is substantially homogeneous in several other aspects. They were all women of Finnish origin, with very similar cultural backgrounds, and they had all suffered acute leukaemia. Sexuality is strongly linked to cultural atmosphere and customs, which may change rapidly, at the behavioural level, among adolescents. ${ }^{20}$ Thus, it is essential for an investigation like this to contain healthy, age matched, and unbiased controls studied during the same period as the survivors. Because the number of subjects in this study is relatively small, particularly the number of sexually experienced subjects, the conclusions should be treated with caution.

To minimise the difficulties of obtaining valid data through interviews with adolescents, the same experienced female adolescent psychiatrist interviewed and assessed the survivors and the controls. In the face to face interview we focused on diminishing the influence of defensive control and overadaptation. ${ }^{12}{ }^{14}$ The limitation of our study stems from its strength: by using a semistructured interview which allows for multiple answers with further questions, it is possible to obtain in depth information that is lost in more structured methods. ${ }^{32-34}$ However, the psychometric properties are not yet known, and comparisons with other cancer populations would be difficult.

We addressed the problem of reliability by using three distinct methods. The reliability and validity of the self report questionnaire used (KISS) have been tested in the Finnish adolescent population $^{21}$ and is very well suited to this population. The findings for the healthy controls under the age of 19 years are similar to those for 17 year old girls examined in 1986 and 1988 in the KISS study. ${ }^{20}{ }^{21}$ The findings about dating, based on both the self report questionnaire and the clinical interview, were identical, which validates the subjects' statements at the interviews.

The interviewing psychiatrist evaluated the subjects after all interviews had been carried out. The interviewer was not acquainted with any of them before the study. However, she could not remain blind to the two groups of subjects since experience of illness was discussed in the interview. Psychiatric evaluations may include a risk of observer bias when interrater was not used. Theoretically, bias may have influenced the evaluations, but it can hardly explain the differences observed. In addition, psychological testing, the Rorschach test in particular, provides a less familiar, less structured setting than do interviews; consequently, the test situation itself facilitates the expression of underlying personality structures and dynamics, ${ }^{35}$ although its reliability as a scientific method is difficult to verify. ${ }^{27}$ In the present study the psychological testing was analysed independently by an experienced psychologist. The findings of the test may include observer bias. However, the material from the interview and the Rorschach test was analysed separately and did not influence each other. Thus, the psychological testing may also serve as an independent rating. The findings of the Rorschach test and the psychiatric evaluations based on the interview were highly correlated with each other, which indicates reliability. Furthermore, the findings based on the questionnaire, the interview, and the Rorschach test were congruent.

Our results indicate that some female survivors have appropriate sexual development after childhood leukaemia. However, the majority of them remain vulnerable with regard to inner sexuality in spite of good adjustment at the 
overt behavioural level. This may lead to subsequent problems with sexuality during adult life. Will the same vulnerability persist or will individual survivors be able to overcome their problems? Recognition of these aspects may help the treatment and follow up of adolescent patients.

We express our gratitude to Professor Jouko Lönnqvist for reviewing the manuscript and for his valuable comments.

This study was supported by the Yriö Jahnsson Foundation, The Finnish Cultural Foundation, and the Finnish Association of Adolescent Psychiatry.

1 Meadows AT, Silber J. Delayed consequences of therapy for childhood. Cancer 1985;35:271-86.

2 Lansky SB, List MA, Ritter-Sterr C. Psychosocial consequences of cure. Cancer 1986;58:529-33.

3 Tope DM, Ahles TA, Silberfarb PM. Psycho-oncology: psyTope DM, Ahles TA, Silberfarb PM. Psycho-oncology: psy-
chosocial well-being as one component of quality of life
Psychother Psychosom 1993;60:129-47.

4 Brown RT, Kaslow NJ, Hazzard AP, et al. Psychiatric and family functioning in children with leukemia and parents. $\mathcal{F}$ Am Acad Child Adolesc Psychiatry 1992;31:495-502.

5 Brown RT, Kaslow NJ, Hazzard AP, et al. Parental psychopathology and children's adjustment to leukemia. $\mathcal{f} A m$ Acad Child Adolesc Psychiatry 1993;32:554-561.

6 Fritz GK, Williams JR, Amylon M. After treatment ends: psychosocial sequelae in pediatric cancer survivors. $A m \mathcal{F}$ Orthopsychiatry 1988:58:552-61.

7 Gray RE, Doan BD, Shermer MA, et al. Psychological adaptation of survivors of childhood cancer. Cancer 1992;70:2713-21.

8 Greenberg HS, Kazak AE, Meadows AT. Psychological functioning in 8- to 16 -year-old cancer survivors and their functioning in 8- to 16-year-old cance

parents. F Pediatr 1989;114:488-93.
9 Fritz GK, Williams JR. Issues of adolescent development for survivors of childhood cancer. $\mathfrak{F}$ Am Acad Child Adolesc survivors of childhood canc
Psychiatry 1988:27:712-5.

10 Koocher GP, O'Malley JE, Gogan JL, Foster DJ. Psychosocial adjustment among paediatric cancer survivors. F Child Psychol Psychiatry 1980;21:163-73.

11 Mulhern RK, Wasserman AL, Friedman AG, Fairclough D. Social competence and behavioural adjustment of children who are long-term survivors of cancer. Pediatrics 1989;83 $18-25$.

12 Wasserman AL, Thompson EI, Wilimas JA, Fairclough DL. The psychological status of survivors of childhood/ adolescent Hodgkin's disease. Am f Dis Child 1987;141: 626-31.

13 Van Dongen Melman JEWM, Sanders-Woustra JAR Psychosocial aspects of childhood cancer: a review of the literature. 7 Child Psychol Psychiatry 1986;27:145-80.

14 Madan-Swain A, Brown RT, Sexson SB, Baldwin K, Pais R, Ragad A. Adolescent cancer survivors, psychosocial and Ragad A. Adolescent cancer survivors, psychosocia

15 Smith K, Ostroff J, Tan C, Lesko L. Alterations in self-perceptions among adolescent cancer survivors. Cancer Invest $1991 ; 9 \cdot 581-8$

16 Ropponen P, Aalberg V, Rautonen J, Kalmari A, Siimes MA. Psychosexual development of adolescent males after malig nancies in childhood. Acta Psychiatr Scand 1990;82:213-8

17 Ropponen P, Siimes MA, Rautonen J, Aalberg V. Psychosexual problems in male childhood malignancy survivors. Acta Psychiatr Scand 1992;85:143-6.

18 Siimes MA, Ropponen P, Aalberg V, Komulainen J, Rautonen J. Prolactinemia in adolescent males surviving malignancies in childhood: impaired dating activity. $\mathcal{f} A d o-$ lesc Health 1993;14:1-5.

19 Gustafsson G, Garwitcz S, Hertz H, et al. A populationbased study of childhood acute lymphoplastic leukemia diagnosed from July 1981 through June 1985 in five Nordic countries: incidence, characteristics, and treatment result. Acta Paediatr Scand 1987;76:781-8.

20 Kontula O. Youth and sexuality: towards independence. In:Lähteenmaa J, Siurala S, eds. Youth and change. Helsinki: In:Lähteenmaa J, Siurala S, eds. Youth and change. Helsinki:
Youth Research Society. Statistics Finland 1992;192:83-9. Youth Research Society. Statistics Finland 1992;192:83-9.
1 Kontula O, Rimpelä M, Ojanlatva A. Sexual knowledge, attitudes, fears and behaviours of adolescents in Finland (the KISS study). Health Education Research 1992;7:69-77.

22 Tirkkonen J, Hukkila K, Kontula O. Tyttöjen ja poikien seksuaalikulttuurit. [Sexual cultures of adolescent girls and boys.] Series original reports 15/89. Publication of Nationa Board of Health, Finland, Helsinki, 1989.

23 Moore BE, Fine BD, eds. Psychoanalytic terms and concepts. New Haven: Yale University Press, 1990.

24 American Psychiatric Association. Diagnostic and statistical manual of mental disorders. 4th Ed (DSM-IV). Washington, DC: American Psychiatric Association, 1994

25 Lerner PM. Psychoanalytic theory and the Rorschach. Hillsdale, NJ: The Analytic Press, 1991.

26 Aronow E, Reaznikoff M. Rorschach content interpretation. Aronow E, Reaznikoff M. Rorschach

27 Klopfer B, Davidson HH. The Rorschach technique. An introductory manual. New York: Harcourt Brace World, 1962.
28 Laufer M, Laufer ME. Adolescence and developmental baufer M, Laufer ME. Adolescence and developmental
breakdown. A psychoanalytic view. Westfird, MA: Yale breakdown. A psych

29 Puukko L-RM, Hirvonen E, Aalberg V, Hovi L, Siimes MA. Impaired body image of young females surviving leukemia. sychosomatics 1997;38:54-62.

30 Blos P. On adolescence. New York: Free Press, 1962.

1 Zani B. Male and female patterns in the discovery of sexuality during adolescence. $\mathcal{F}$ Adolesc 1991;14:163-78.

32 Cappelli M, McGrath PJ, Heick CA, McDonald NE, Feldman W, Rowe P. Chronic disease and its impact: the adolescent's perspective. F Adolesc Health Care 1989;10: 283-8.

33 Kaplan HI, Sadock BJ, eds. Comprehensive textbook of psychiatry $V$. 5th Ed. Baltimore: Williams and Wilkins, 1989.

34 Puukko L-R, Sammallaht PR, Siimes MA, Aalberg VA. Childhood leukemia and body image: interview reveals impairment not found with a questionnaire. 7 Clin Psychol 1997;53:1-5.

35 Lerner HD, Lerner PM, eds. Primitive mental states and the Rorschach. Madison, CT: International Universities Press, 1988

\section{Appendix}

QUESTIONS CONCERNING SEXUAL MATTERS

ASKED AT THE INTERVIEW

1. Have you ever dated?

2 . How old you were when you began dating for the first time?

3. Would you like to date?

4. What do you think about boys/men?

5. How many times have you dated?

6. How old were you when you had sexual intercourse for the first time?

7. With whom?

8. Did you want intercourse yourself?

9. In what kind of circumstances did it take place?

10. Had you taken alcohol?

11. How much? Were you drunk?

12. How did you feel about the first time you had sexual intercourse?

13. The first time can be painful; did you feel any pain?

14. Have you had sexual experiences since then? How often?

15. Do you think your female friends have experienced sexual intercourse?

16. Quite often sexual experiences are not either enjoyable or satisfactory. How about you?

17. How often do you have sexual intercourse? Who is the initiator? Have you ever been the initiator? How often?

18. Do you feel free to tell your partner your desires?

19. Orgasm means that sexual excitement ends in a climax, followed by pleasurable or enjoyable relaxation. Have you experienced orgasm?

20. Sexual intercourse is possible without orgasm. Have you experienced orgasm in sexual intercourse? If yes, how often?

21 . Masturbation is very common, especially among young people or those with no regular sexual partner. Masturbation means that a person caresses her(his)self, which arouses excitement. What do you think about masturbation?

22. Have you ever masturbated?

23. What do you think about homosexuality?

24 . How significant is sexuality in an adult person's life? 